\title{
TERAPIA DE REPOSIÇÃO ENZIMÁTICA PARA AS MUCOPOLISSACARIDOSES I, II E VI: RECOMENDAÇ̃̃ES DE UM GRUPO DE ESPECIALISTAS BRASILEIROS
}

\author{
Roberto Giugliani ${ }^{1 *}$, Andressa Federhen ${ }^{2}$, Maria Verónica Muñoz Rojas ${ }^{3}$, Taiane Alves Vieira ${ }^{4}$, Osvaldo Artigalás 5 , Louise Lapagesse Carmargo Pinto ${ }^{6}$, Ana Cecília \\ Azevedo $^{7}$, Angelina Xavier Acosta ${ }^{8}$, Carmem Bomfim ${ }^{9}$, Charles Marques Lourenço ${ }^{10}$, Chong Ae Kim $^{11}$, Dafne Horovitz ${ }^{12}$, Denize Bomfim Souza ${ }^{13}$, Denise Norato ${ }^{14}$, \\ Diane Marinho ${ }^{15}$, Durval Palhares ${ }^{16}$, Emerson Santana Santos ${ }^{17}$, Erlane Ribeiro ${ }^{18}$, Eugênia Ribeiro Valadares ${ }^{19}$, Fábio Guarany ${ }^{20}$, Gisele Rosone de Lucca ${ }^{21}$, Helena \\ Pimentel ${ }^{22}$, Isabel Neves de Souza ${ }^{23}$, Jordão Corrêa Neto ${ }^{24}$, José Carlos Fraga ${ }^{25}$, José Eduardo Góes ${ }^{26}$, José Maria Cabral ${ }^{27}$, José Simeonato ${ }^{28}$, Juan Clinton Llerena \\ $J_{R .}{ }^{29}$, Laura Bannach Jardim ${ }^{30}$, Liane de Rosso Giulian ${ }^{31}$, Luiz Carlos Santana da Silva ${ }^{32}$, Mara Santos ${ }^{33}$, Maria Ângela Moreira ${ }^{34}$, Marcelo Kerstenetzky ${ }^{35}$, Márcia \\ Ribeiro ${ }^{36}$, Nicole Ruas ${ }^{37}$, Patricia Barrios ${ }^{38}$, Paulo Aranda ${ }^{39}$, Rachel Honjo ${ }^{40}$, Raquel Boy ${ }^{41}$, Ronaldo Costa ${ }^{42}$, Carolina Fishinger Moura de Souza ${ }^{43}$, Flavio $F$ \\ Alcântara ${ }^{44}$, Sylvio Gilberto A. Avilla ${ }^{45}$, Simone Fagondes ${ }^{46}$, Ana Maria Martins ${ }^{47}$
}

Trabalho realizado na Rede MPS Brasil, com sede no Serviço de Genética Médica do Hospital de Clínicas de Porto Alegre, Centro Colaborador da OMS para o Desenvolvimento de Serviços de Genética Médica na América Latina, Porto Alegre, RS.

\section{*Correspondência:} Serviço de Genética Médica, Hospital de Clínicas de Porto Alegre, Rua Ramiro Barcelos 2350 CEP: 90035-903 Porto Alegre - RS

\section{Resumo}

As mucopolissacaridoses (MPS) são doenças genéticas raras causadas pela deficiência de enzimas lisossômicas específicas que afetam o catabolismo de glicosaminoglicanos (GAG). 0 acúmulo de GAG em vários órgãos e tecidos nos pacientes afetados pelas MPS resulta em uma série de sinais e sintomas, integrantes de um quadro clínico multissistêmico que compromete ossos e articulações, vias respiratórias, sistema cardiovascular e muitos outros órgãos e tecidos, incluindo, em alguns casos, as funções cognitivas. Já foram identificados 11 defeitos enzimáticos que causam sete tipos diferentes de MPS. Antes do advento de terapias dirigidas para a restauração da atividade da enzima deficiente, o tratamento das MPS tinha como principal foco a prevenção e o cuidado das complicações, aspecto ainda bastante importante no manejo desses pacientes. Na década de 80 foi proposto o tratamento das MPS com transplante de medula óssea/transplante de células tronco hematopoiéticas (TMO/TCTH) e na década de 90 começou o desenvolvimento da Terapia de Reposição Enzimática (TRE), que se tornou uma realidade aprovada para uso clínico nas MPS I, II e VI na primeira década do século 21. Os autores deste trabalho têm a convicção de que um melhor futuro para os pacientes afetados pelas MPS depende da identificação, compreensão e manejo adequado das manifestações multissistêmicas dessas doenças, incluindo medidas de suporte (que devem fazer parte da assistência multidisciplinar regular destes pacientes) e terapias específicas. Embora a inibição da síntese de GAG e o resgate da atividade enzimática com moléculas pequenas também possam vir a ter um papel no manejo das MPS, o grande avanço disponível no momento é a TRE intravenosa. A TRE permitiu modificar radicalmente o panorama do tratamento das mucopolissacaridoses I, II e VI na última década, sendo que ainda pode estender seus benefícios em breve para a MPS IV A (cuja TRE já está em desenvolvimento clínico), com perspectivas para o tratamento da MPS III A e do déficit cognitivo na MPS II através de administração da enzima diretamente no sistema nervoso central (SNC). Um grande número de centros brasileiros, incluindo serviços de todas as regiões do país, já têm experiência com TRE para MPS I, II e VI. Essa experiência foi adquirida não só com o tratamento de pacientes como também com a participação de alguns grupos em ensaios clínicos envolvendo TRE para essas condições. Somados os três tipos de MPS, mais de 250 pacientes já foram tratados com TRE em nosso país. A experiência dos profissionais brasileiros, somada aos dados disponíveis na literatura internacional, permitiu elaborar este documento, produzido com o objetivo de reunir e harmonizar as informações disponíveis sobre o tratamento destas doenças graves e progressivas, mas que, felizmente, são hoje tratáveis, uma realidade que traz novas perspectivas para os pacientes brasileiros afetados por essas condições.

UnITERMOS: Mucopolissacaridoses. Mucopolissacaridose I. Mucopolissacaridose II. Mucopolissacaridose VI. Glicosaminoglicanos. Terapia de reposição enzimática.

1. Médico, Doutor em Genética - Professor Titular do Departamento de Genética da Universidade Federal do Rio Grande do Sul - UFRGS; Diretor, Centro Colaborador da OMS para o Desenvolvimento de Serviços de Genética Médica na América Latina e Coordenador, Instituto Nacional de Genética Médica Populacional INAGEMP, Porto Alegre, RS

2. Enfermeira - Pós-graduada do programa de pós-graduação em ciências médicas da Universidade Federal do Rio Grande do Sul - Coordenadora de pesquisa clínica Grupo de Pesquisa Clínica em Genética Médica, Hospital das Clínicas de Porto Alegre, RS

3. Médica, Mestre em Genética - Pós-graduanda, Programa de Pós-Graduação em Saúde da Criança e do Adolescente da Universidade Federal do Rio Grande do Sul UFRGS, Porto Alegre, RS

4. Enfermeira-Mestre em Ciências Médicas - Pós-graduada do programa de pós-graduação em ciências médicas da Universidade Federal do Rio Grande do Sul Coordenadora de pesquisa clínica, Grupo de Pesquisa Clínica em Genética Médica, Hospital de Clínicas de Porto Alegre, RS 
5. Médico, Mestre em Genética e Biologia Molecular - Médico Geneticista do Grupo Hospitalar Conceição, Porto Alegre, RS

6. Médica, Doutora em Saúde da Criança e do Adolescente - Médica do Grupo de Pesquisa Clínica em Genética Médica, Hospital de Clínicas de Porto Alegre, RS

7. Médica, Doutora em Saúde da Criança e do Adolescente - Médica da Prefeitura Municipal de Gravataí, RS

8. Médica, Doutora em Genética - Professora Adjunta do Departamento de Pediatria da Universidade Federal da Bahia, Salvador, BA

9. Médica - Serviço de Transplante de Medula Óssea, Hospital de Clínicas, Universidade Federal do Paraná, Curitiba, PR

10. Médico - Pós-graduando, Setor de Neurogenética, Faculdade de Medicina de Ribeirão Preto da Universidade de São Paulo - USP, Ribeirão Preto, SP

11. Médica, Livre-Docente - Chefe, Unidade de Genética, Instituto da Criança, Hospital de Clínicas, Faculdade de Medicina da Universidade de São Paulo - USP, São Paulo, SP

12. Médica, Doutora - Médica Geneticista do Centro de Genética Médica, Instituto Fernandes Figueira, FIOCRUZ, Rio de Janeiro, RJ

13. Médica - Neurologista Infantil, Hospital Universitário, Brasília, DF

14. Doutora - Assessora, Faculdade de Medicina - PUC, Campinas, SP

15. Médica - Serviço de Oftalmologia, Hospital de Clínicas de Porto Alegre, Porto Alegre, RS

16. Doutor - Professor Titular, Departamento de Pediatria, Universidade Federal do Mato Grosso do Sul, Campo Grande, MS

17. Médico - Professor, Universidade Estadual de Ciências da Saúde de Alagoas, Maceió, AL

18. Mestre - Professora Titular, Faculdade de Medicina de Juazeiro do Norte, CE

19. Doutora - Professora Adjunta, Departamento de Pediatria da Universidade Federal de Minas Gerais - UFMG, Belo Horizonte, MG

20. Médico- Serviço de Fisiatria, Hospital de Clínicas de Porto Alegre, Porto Alegre, RS

21. Médica - Hospital Infantil Joana de Gusmão, Florianópolis, SC

22. Médica - APAE, Salvador, BA

23. Mestre - Professora Assistente da Universidade Federal do Pará, Belém, PA

24. Mestre - Médico geneticista, Hospital do Servidor Público Estadual, São Paulo, SP

25. Doutor - Professor Adjunto, Departamento de Cirurgia pela Universidade Federal do Rio Grande do Sul - UFRGS, Porto Alegre, RS

26. Médico, Hospital Infantil Joana de Gusmão, Florianópolis, SC

27. Mestre - Professor Titular, Universidade Federal do Amazonas, Manaus, AM

28. Médico, Hospital Infantil, Belo Horizonte, MG

29. Doutor-Chefe, Centro de Genética Médica, Instituto Fernandes Figueira, FIOCRUZ, Rio de Janeiro, RJ

30. Doutora - Professora Adjunta, Departamento de Medicina Interna da Universidade Federal do Rio Grande do Sul - UFRGS, Porto Alegre, RS

31. Mestre - Professora Assistente, Departamento de Pediatria da Universidade Federal do Mato Grosso do Sul - UFMS, Campo Grande, MS

32. Doutor - Professor Associado, Instituto de Ciências Biológicas, Universidade Federal do Pará - UFPA, Belém, PA

33. Especialista em Neuropediatria - Médica, Hospital Infantil Pequeno Príncipe, Curitiba, PR

34. Doutora - Médica, Serviço de Pneumologia, Hospital de Clínicas de Porto Alegre, Porto Alegre, RS

35. Médico - Hospital da Restauração, Recife, PE

36. Doutora - Professora Adjunta, Departamento de Pediatria da Universidade Federal do Rio de Janeiro - UFRJ, Rio de Janeiro, RJ

37. Terapeuta Ocupacional, Hospital de Clínicas de Porto Alegre, Porto Alegre, RS

38. Doutorado - Cardiologista Infantil, Serviço de Cardiologia, Hospital de Clínicas de Porto Alegre, Porto Alegre, RS

39. Médico, Hospital Evangélico, Londrina, PR

40. Mestre - Médica, Instituto de Criança, Faculdade de Medicina da Universidade de São Paulo - USP, São Paulo, SP

41. Médica, Mestre em Saúde da Criança - Professora Assistente, Universidade Estadual do Rio de Janeiro, Rio de Janeiro, RJ

42. Médico - Anestesiologista, Hospital de Clínicas de Porto Alegre, Porto Alegre, RS

43. Médica, Doutora em Genética - Representante da Sociedade Brasileira de Genética Médica, São Paulo, SP

44. Médico, Patologista Clínico - Representante da Sociedade Brasileira de Patologia Clínica, São Paulo, SP

45. Médico, Cirurgião Pediátrico - Representante da Sociedade Brasileira de Cirurgia Pediátrica, São Paulo, SP

46. Médica, Doutora - Representante da Sociedade Brasileira de Pneumologia e Tisiologia, Brasília, DF

47. Médica, Doutora - Professora Adjunta, Departamento de Pediatria, Universidade Federal de São Paulo - UNIFESP, São Paulo, SP

\section{INTRODUÇÃO}

As mucopolissacaridoses (MPS) são doenças genéticas raras causadas pela deficiência de enzimas lisossômicas específicas que afetam o catabolismo de glicosaminoglicanos (GAG). O acúmulo de GAG em vários órgãos e tecidos nos pacientes afetados pelas MPS resulta em uma série de sinais e sintomas, integrantes de um quadro clínico multissistêmico. Já foram identificados 11 defeitos enzimáticos que causam sete tipos diferentes de MPS ${ }^{1}$.

As manifestações mais frequentes das MPS incluem principalmente fácies característica, opacificação da córnea e/ou outros problemas oculares, macroglossia, perda auditiva, hidrocefalia, cardiopatia, problemas respiratórios, hepatoesplenomegalia, hérnia inguinal e umbilical, disostose múltipla, limitação da mobilidade articular e dano cognitivo (em parte dos casos) ${ }^{1}$. Devido ao comprometimento de vários órgãos e tecidos, os pacientes com MPS frequentemente necessitam de intervenções cirúrgicas que podem levar a um alto índice de complicações².

A suspeita clínica de uma MPS é motivo para realizar a dosagem dos GAG urinários. Estes estão quantitativamente elevados em praticamente todos os casos de MPS, embora a ocorrência de níveis normais não permita descartar o diagnóstico em paciente com quadro clínico sugestivo. A cromatografia ou eletroforese podem ser usadas para identificar qual o tipo de GAG em excesso (dermatan-sulfato, heparan-sulfato, queratansulfato, por exemplo), auxiliando a definir quais enzimas deverão ser inicialmente testadas ${ }^{3}$. O diagnóstico das MPS deve ser confirmado através de ensaio enzimático, documentando a atividade deficiente da enzima específica para cada tipo de MPS.

A identificação do genótipo pode ser importante para prever o fenótipo (e para decisões terapêuticas em alguns casos), para possibilitar o aconselhamento genético na família e para auxiliar no diagnóstico pré-natal.

Antes do advento de terapias dirigidas para a restauração da atividade da enzima deficiente, o tratamento das MPS I, II e VI tinha como principal foco a prevenção e o cuidado das complicações, aspecto ainda bastante importante no manejo desses pacientes.

Na década de 80 foi proposto o tratamento das MPS com transplante de medula óssea/transplante de células tronco hematopoiéticas 
(TMO/TCTH $)^{4,5}$, que ainda hoje é recomendado para pacientes com a forma grave de MPS I diagnosticados antes dos dois anos de idade, pela possibilidade desta terapia prevenir ou retardar o dano cognitivo. De uma maneira geral, o TMO/TCTH não tem sido recomendado para as demais formas de MPS I e para os pacientes com MPS VI (pelos altos riscos associados, o que o torna de indicação questionável em função da existência de terapia enzimática), nem para MPS II (em função da ausência de bons resultados).

Na década de 90 começou o desenvolvimento da Terapia de Reposição Enzimática (TRE), que se tornou uma realidade aprovada para uso clínico em 2003 para MPS I, em 2005 para MPS VI e em 2006 para MPS $\|^{6-19}$.

Um grupo de profissionais brasileiros com experiência no tratamento de MPS, representando diversas especialidades e todas as regiões do país, se reuniu para elaborar as diretrizes para o tratamento das MPS I, II e VI, doenças para as quais já há uma terapia específica.

\section{Terapia de reposição enzimática para mucopolissacaridoses}

A terapia de reposição enzimática (TRE) é um tratamento que consiste na administração periódica, por via venosa, da enzima específica deficiente no paciente. A realização do primeiro tratamento eficaz com TRE em pacientes com a doença de Gaucher ${ }^{20}$ levou à busca de tratamento semelhante para outras doenças de depósito lisossômico. A primeira TRE para tratamento de uma mucopolissacaridose foi a para MPS $\left.\right|^{21}$, sendo subsequentemente aprovadas as TRE para MPS VI ${ }^{22}$ e para MPS $\|^{23}$.

A TRE para MPS I é realizada pela administração intravenosa de laronidase, uma proteína análoga à a-iduronidase humana produzida por engenharia genética num sistema de expressão de células de ovário de hamster chinês $(\mathrm{CHO})^{4}$. A TRE com laronidase foi aprovada para o tratamento de pacientes nos Estados Unidos e na Europa em $2003^{24,25}$ e no Brasil em $2005^{26}$. A TRE para o tratamento de MPS II é realizada pela administração intravenosa de idursulfase, uma proteína glicosilada análoga à iduronato-2-sulfatase humana nativa, produzida através de engenharia genética em uma linhagem continua de células humanas ${ }^{59}$. A TRE com idursulfase foi aprovada para o tratamento de pacientes nos Estados Unidos em julho de $2006^{82}$ e na Europa em janeiro de $2007^{83}$, tendo o registro na ANVISA ocorrido em $2008^{84}$. A TRE para o tratamento de MPS VI é realizada pela administração intravenosa de galsulfase, uma forma recombinante da enzima $\mathrm{N}$-acetilgalactosamina 4-sulfatase, sintetizada através de engenharia genética a partir de células de ovário de hamster chinês ${ }^{17,27,28}$. A comercialização e uso da galsulfase foi aprovada nos Estados Unidos em $2005^{24}$, na Comunidade Européia em janeiro 2006, ${ }^{25}$ e em fevereiro de 2009 no Brasil, quando recebeu o registro da ANVISA ${ }^{26}$.

\section{Indicações para o uso de TRE}

MPS I - De forma objetiva, a TRE para MPS I está indicada para pacientes de qualquer idade, que tenham o diagnóstico confirmado, que sejam sintomáticos, e que apresentem pelo menos uma manifestação clínica que responda ao tratamento com TRE. Estas manifestações podem ser: a) doenças respiratórias tais como obstruções de vias áreas superiores, infecção de repetição, doenças restritivas; b) comprometimento cardíaco como miocardiopatia e doença valvar; c) comprometimento osteoarticular que prejudique ou dificulte a locomoção e torne o paciente dependente de outras pessoas para a realização de atividades diárias; d) apneia do sono com índice de apnéias (IA) maior que 1 evento/hora de sono para pacientes menores de 17 anos e maior que 5 eventos/hora de sono para adultos; e) saturação média de 02noturno < 92\% em crianças e < 85\% em adultos; f) pacientes de difícil intubação.

MPS II - A TRE para pacientes com MPS II está usualmente indicada para todos os pacientes sintomáticos com diagnóstico confirmado de MPS II. Embora Wraith et al. ${ }^{29}$ sugiram que mesmo pacientes com importante envolvimento do Sistema Nervoso Central (SNC) devem receber TRE por 12 a 18 meses, cuja manutenção deveria ser avaliada após esse período, os benefícios globais do tratamento são questionáveis em pacientes que apresentam grave comprometimento das funções cognitivas, já que a enzima administrada por via intravenosa não atravessa a barreira hematoencefálica. De forma objetiva, a TRE deve ser indicada para pacientes de qualquer idade que sejam sintomáticos e que apresentem pelo menos uma manifestação clínica que responda ao tratamento com TRE, tais como: a) Doenças respiratórias como obstruções de vias áreas superiores, infecções de repetição, doenças restritivas; b) Comprometimento osteoarticular que prejudique ou dificulte a locomoção e torne o paciente dependente de outras pessoas para a realização de atividades diárias; c) Apneia do sono com frequência maior que 1 evento/hora para pacientes menores de 18 anos e maior que 5 eventos/hora para adultos; d) Saturação média de 02noturno $<92 \%$ em crianças e $<85 \%$ em adultos.

MPS VI - A TRE pode ser administrada a todos os pacientes sintomáticos com diagnóstico de MPS VI confirmado, sendo recomendável como primeira escolha terapêutica. Estudos demonstraram melhora no teste da caminhada e na capacidade de subir escadas ${ }^{30,31}$ e melhora na doença óssea relacionada a MPS VI, assim como melhora no padrão de crescimento em um paciente tratado a partir da oitava semana de vida ${ }^{32}$. Sabe-se, entretanto, que alguns tecidos como a córnea, por sua menor perfusão, e o SNC, pela barreira hematoencefálica, não são atingidos de forma significativa pela ação da enzima administrada por via intravenosa ${ }^{16,30}$. De forma objetiva, a TRE deve ser indicada para pacientes de qualquer idade e que sejam sintomáticos, e que apresentem pelo menos uma manifestação clínica que responda ao tratamento com TRE. Estas manifestações podem ser: a) Doenças respiratórias tais como obstruções de vias áreas superiores, infecções de repetição, doenças restritivas; b) Comprometimento osteoarticular que prejudique ou dificulte a locomoção e torne o paciente dependente de outras pessoas para a realização de atividades diárias; c) Apneia do sono com frequência maior que 1 evento/hora para pacientes menores de 18 anos e maior que 5 eventos/hora para adultos; d) Saturação média de 02 noturno $<92 \%$ em crianças e $<85 \%$ em adultos; e) Pacientes de difícil intubação.

\section{Informações adicionais sobre a TRE nas MPS I, II e VI}

Sobre o regime de uso - É recomendado o uso da dose padrão. Alguns pequenos ajustes podem ser feitos de modo que a dose utilizada varie não mais do que $10 \%$ em relação à dose padrão. A dose mensal final também não deve variar mais do que $10 \%$ daquela que seria a dose mensal ideal estabelecida de acordo com 
a dose padrão. Recomenda-se que a infusão seja inicialmente realizada no ambiente hospitalar e, de preferência, em ambiente Iúdico e agradável para o paciente. Face ao número crescente de pacientes em todo o país que recebem TRE, recomenda-se que esse procedimento seja normatizado dentro do SUS, passando a ser um dos procedimentos oficialmente contemplados para ser realizado em regime de "hospital-dia". É importante haver alternância dos sítios de punção venosa periférica. Quando utilizar cateter central totalmente implantado, recomenda-se utilização de EMLA® (1h ou 1h30min pré-punção). Recomenda-se que o paciente seja observado por, pelo menos, uma hora após o término da infusão, no decorrer, pelo menos, dos primeiros três meses de tratamento, se não for possível pelo período ideal que é de seis meses. Após esse período de observação, e não havendo qualquer fator complicador, o paciente poderá ser liberado imediatamente após a infusão. Um estudo recente em MPS I19 indicou que a utilização do dobro da dose a cada duas semanas não resulta em desvantagens significativas para o paciente, podendo este regime de administração ser considerado (o estudo se refere apenas a MPS I) nos casos em que a aplicação de um regime de infusão semanal seja, por alguma razão operacional ou logística, difícil de ser realizada. Uma orientação geral sobre o regime de uso das medicações para TRE para MPS I, II e VI está disponível na Tabela I.

Contra-indicações - A TRE não é indicada para mulheres grávidas ou que estejam amamentando, a não ser que seja absolutamente indispensável. Pacientes terminais também não devem receber TRE e nem aqueles portadores de uma doença grave associada, cujo prognóstico não se alterará devido ao uso da TRE.

Pré-medicação - As eventuais reações à infusão são muito particulares para cada paciente, de modo que o médico deve avaliar a necessidade da pré-medicação e a sua intensidade caso

\section{Tabela 1 - Terapia de reposição enzimática para MPS I, II e VI:} regime de uso (dose, periodicidade e tempo de infusão)

\section{MPS I}

Medicamento e fabricante

Apresentação

Dose padrão e via de administração

Periodicidade

Tempo de infusão

Aldurazyme $^{\circledR}$ (Genzyme Corporation)
Frascos com 2,9 mg/5mL $(0,58 \mathrm{mg} / \mathrm{mL})$
$0,58 \mathrm{mg} / \mathrm{kg}$, via intravenosa
Semanal ( $7+$ - 3 dias)
Aproximadamente $3-4$ horas

\section{MPS II}

Medicamento e fabricante

Apresentação

Dose padrão e via de administração

Periodicidade

Tempo de infusão

MPS VI

Medicamento e fabricante

Apresentação

Dose padrão e via de administração

Periodicidade

Tempo de infusão a caso. A pré-medicação com antipiréticos e/ou anti-histamínicos é, geralmente, utilizada na TRE de pacientes com MPS I. No caso da TRE em pacientes com MPS VI, têm sido utilizados anti-histamínicos, com ou sem antipiréticos, cerca de uma hora antes do início da infusão. Se houver reação à infusão, que persista mesmo com uso de antipiréticos e anti-histamínicos, deve ser considerado o uso de corticoesteróides antes da TRE, por exemplo, predinisolona ( $1 \mathrm{mg} / \mathrm{kg}$ ) 12 horas e uma hora antes da infusão. Não é prescrito o uso de pré-medicação, como rotina, na TRE de pacientes com MPS II, a não ser para prevenção de recorrência de reações à infusão.

Preparação da medicação - Utilizando técnicas adequadas de assepsia, a medicação deve ser preparada como indicado: a) Determinar o número de frascos a serem diluídos, com base no peso do paciente e na dose padrão recomendada da enzima de reposição, ajustando de tal forma que sejam utilizados frascos completos; b) Retirar os frascos da geladeira para que alcancem a temperatura ambiente. Esses frascos não devem ser aquecidos; c) Observar o aspecto, uma vez que a solução é transparente ou discretamente amarelada, e clara ou levemente opalescente. Se a solução apresentar características alteradas, recomenda-se não utilizar estes frascos; d) Determinar o volume final total a ser infundido, que depende do peso do paciente e da medicação a ser preparada: MPS I: $100 \mathrm{~mL}$ (peso $\leq$ a $20 \mathrm{~kg}$ ) ou $250 \mathrm{~mL}$ (peso > $20 \mathrm{~kg}$ ); MPS II: $100 \mathrm{~mL}$ (para todos os pesos); MPS VI: $250 \mathrm{~mL}$ (em geral - para peso igual ou inferior a $20 \mathrm{~kg}$; em pacientes com susceptibilidade à sobrecarga de volume, o médico pode considerar o volume total de $100 \mathrm{~mL}$ ); e) Aspirar lentamente o volume de enzima calculado dos frascos, cuidando para não agitar a solução, pois a agitação pode desnaturar o produto e torná-lo biologicamente inativo; f) Retirar da bolsa de soro fisiológico correspondente (100 $\mathrm{mL}$ ou $250 \mathrm{~mL}$ ) um volume igual àquele calculado e retirado dos frascos de enzima, de tal forma que ao acrescentar o volume de enzima, seja reconstituído um volume final total igual a $100 \mathrm{~mL}$ ou 250 mL; o acréscimo da solução de enzima na bolsa de soro fisiológico deve ser lento e a bolsa com a solução final deverá ser levemente movimentada para permitir a distribuição homogênea da medicação. Esta solução deverá ser usada imediatamente. Se o uso imediato não for possível, a solução deverá ser armazenada sob refrigeração $\left(2^{\circ} \mathrm{C}\right.$ a $\left.8^{\circ} \mathrm{C}\right)$ por um período máximo de 36 horas desde o preparo até o final da administração da mesma. Não deixe a solução preparada à temperatura ambiente; g) No caso da MPS I, o uso de albumina é recomendado nos Estados Unidos, mas não na Europa. No Brasil, a bula aprovada pela ANVISA recomenda o uso de albumina, mas a experiência dos especialistas brasileiros indica que isso é prescindível.

Velocidade de infusão - Após o preparo da medicação, a infusão deverá ser administrada preferencialmente em bomba de infusão de forma escalonada, conforme recomendado. No entanto, em caso de reações associadas à infusão, as velocidades de escalonamento e velocidade final máxima alcançada poderão ser modificadas de acordo com a tolerabilidade de cada paciente.

Uso de Filtros - Recomenda-se que a administração da solução de laronidase, idursulfase e galsulfase, seja realizada utilizando um equipo de infusão com filtro de 0,2 $\mu \mathrm{m}$.

Reações adversas - As reações à infusão mais frequentemente relatadas com uso de TRE foram: pirexia, cefaleia, dor abdominal, dispneia, calafrios, artralgia, prurido, hipertensão/ 
hipotensão, urticária e exantema (rash). Se uma reação à infusão ocorrer, tendo ou não havido uso de pré-medicação, as seguintes medidas devem ser tomadas, nesta ordem, até que melhorem os sintomas: a) Diminuição da velocidade de infusão; b) Interrupção temporária da infusão; c) Administração adicional de antipiréticos e anti-histamínicos. Se uma reação de hipersensibilidade grave ocorrer, ou uma reação anafilática, a infusão deve ser interrompida imediatamente e as medidas de suporte adequadas devem ser estabelecidas prontamente de acordo com o quadro apresentado. Pode ser necessário o uso de corticoesteroides e de medidas de manutenção de vias aéreas e de acesso venoso. Medidas de ressuscitação devem ser implementadas em casos extremos. Por este motivo, recomenda-se que o centro de infusão esteja equipado com material necessário para assistência integral à parada cardiorrespiratória (carro de parada) e tenha fácil acesso à sala de emergência. Caso seja considerado o uso de epinefrina, este uso deve ser realizado com extrema cautela devido à prevalência aumentada de doença coronariana em muitos pacientes com MPS. A relação risco/benefício da administração da enzima após uma reação de hipersensibilidade grave ou reação anafilática deve ser ponderada e, se as infusões de TRE forem reiniciadas, medidas apropriadas de ressuscitação devem estar disponíveis para utilização caso seja necessário. Idealmente, antes de iniciar a TRE, deve ser feita uma coleta de sangue para dosagem de anticorpos. Esta amostra será guardada até que esta dosagem seja necessária, isto é, em caso do paciente vir a apresentar uma reação à infusão. Se o paciente apresentar uma reação à infusão, uma nova coleta de sangue deverá ser realizada entre 1 e 2 horas do início da reação, ou de acordo com as orientações do fabricante da enzima. Qualquer efeito colateral deve ser comunicado, o mais prontamente possível, ao Departamento de Farmacovigilância da ANVISA e ao setor de farmacovigilância do hospital, quando aplicável. Além disto, as empresas responsáveis pelos medicamentos laronidase (Genzyme), idursulfase (Shire/HGT) e galsulfase (BioMarin), solicitam que sejam notificadas através de seus departamentos médicos, para fins de farmacovigilância.

Rotina clínica - Antes do início de cada infusão devem ser realizados breve anamnese e exame físico dirigido, com verificação dos sinais vitais. Eventualmente, poderá ser indicada a coleta de amostras para exames de acompanhamento. Os pacientes não precisam estar em jejum e nem a alimentação dos pacientes precisa ser modificada em virtude da infusão.

Critérios para interrupção do tratamento - Até o momento não há critérios estabelecidos determinando a indicação de interrupção do tratamento, porém recomenda-se que a TRE seja interrompida: a) Durante a gestação e a amamentação; b) No paciente que, apesar da TRE, evoluir para doença terminal ou que apresente piora significativa nos seus parâmetros clínicos, mensurados no mínimo a cada seis meses e, preferencialmente por um período não inferior a 12 meses de TRE; c) Paciente que não apresente benefício clínico mensurável, levando em consideração o ritmo de progressão natural da doença, através de parâmetros mensurados no mínimo a cada seis meses e, preferencialmente por um período não inferior a 12 meses de TRE. A possibilidade de interrupção do tratamento deve ser apresentada aos pais/paciente ou responsáveis quando a TRE estiver sendo considerada e antes de iniciá-la. Durante o acompanhamento clínico do paciente em TRE, os parâmetros de resposta terapêutica à TRE deverão ser avaliados periodicamente e discutidos com os pais/paciente ou responsáveis. Se a interrupção da TRE estiver sendo considerada, esta deve ser discutida com os pais/paciente ou responsável. Quando a suspensão temporária da TRE estiver sendo considerada, deve-se levar em conta que os poucos relatos de experiência com interrupção da TRE encontrados na literatura mostram que a descontinuidade do tratamento pode levar a uma rápida deterioração do quadro clínico do paciente ${ }^{33,34}$.

Tratamento pré-sintomático - Embora existam resultados bastante encorajadores, os benefícios do tratamento pré-sintomático observados em diversos relatos de casos ainda não foram aferidos por ensaios clínicos (que já estão em andamento no caso da MPS VI). Deste modo, o tratamento da MPS I, MPS II e MPS $\mathrm{VI}$ antes do aparecimento dos sintomas deve ser apresentado à família, nos casos em que o médico achar indicado, como um procedimento experimental, sugerindo-se a aplicação de Termo de Consentimento Livre e Esclarecido aprovado pelas instâncias éticas competentes.

Tratamento em crianças pequenas - O uso de laronidase em crianças com menos de cinco anos se mostrou seguro, conforme demonstrado em estudo clínico específico em crianças pequenas como MPS I $^{15}$. Essa experiência favorável em termos de segurança também foi observada de maneira consistente em inúmeros casos de MPS II e MPS VI tratados com TRE ${ }^{35}$, embora ainda não tenha sido formalmente aferida por ensaios clínicos realizados em crianças pequenas (há um estudo clínico em andamento no caso da MPS II).

Vias alternativas de administração - O Brasil foi pioneiro na administração intratecal da enzima recombinante em um paciente com MPS I, para tratamento da compressão medular. Essa experiência teve resultados animadores e foi relatada na literatura ${ }^{36}$. Casos adicionais de pacientes brasileiros com MPS e compressão medular (um de MPS I e outro de MPS VI) foram tratados dessa forma e os relatos estão sendo preparados para publicação. A administração da enzima por via intratecal ainda deve ser considerada, neste momento, um procedimento experimental.

Infusão domiciliar - A infusão domiciliar pode se constituir numa opção para os pacientes que, após três a seis meses de infusão hospitalar, não tenham apresentado reações significativas à infusão. É recomendado que o local da infusão e também - local de armazenamento e preparo da medicação sejam aprovados pelo responsável pela equipe médica do centro de referência, e que um profissional de enfermagem treinado nesse procedimento específico acompanhe a infusão durante todo o tempo e informe regularmente ao centro de referência sobre o procedimento. 0 paciente em infusão domiciliar deve fazer suas revisões médicas regulares no centro de referência, no mínimo a cada três meses ${ }^{37}$.

\section{Perspectivas e conclusões}

Os autores deste trabalho têm a convicção de que um melhor futuro para os pacientes afetados pelas mucopolissacaridoses depende da identificação, compreensão e manejo adequado das manifestações multissistêmicas dessas doenças, incluindo medidas de suporte (que devem fazer parte da assistência multidisciplinar regular destes pacientes) e terapias específicas. Existem indicações de que a detecção mais precoce dos casos, eventualmente através de triagem neonatal, possa vir a contribuir 
para um melhor prognóstico. Uma cura definitiva talvez seja alcançada através da terapia gênica, mas há ainda um longo caminho a percorrer até esse momento.

Embora a inibição da síntese de glicosaminoglicanos e o resgate da atividade enzimática com moléculas pequenas também possam vir a ter um papel no manejo das MPS, o grande avanço disponível no momento é a TRE. Ao lado do TMO/TCTH (para situações específicas), a TRE permitiu modificar radicalmente 0 panorama do tratamento das mucopolissacaridoses I, II e VI na última década e vem ajudando na melhor compreensão da fisiopatologia da doença ${ }^{38}$ e de potenciais biomarcadores ${ }^{39}$, podendo ainda estender seus benefícios para a MPS IV A (cuja TRE está em desenvolvimento clínico) num futuro não muito distante, com perspectivas para o tratamento da MPS III A e do déficit cognitivo na MPS II através de administração da enzima diretamente no SNC.

Um grande número de centros brasileiros, incluindo serviços de todas as regiões do país, já têm experiência com TRE para MPS I, II e VI. Essa experiência foi adquirida não só com o tratamento de pacientes como também com a participação de alguns grupos em ensaios clínicos envolvendo TRE para essas condições. Somados os três tipos de MPS, mais de 200 pacientes foram tratados com TRE em nosso país. A experiência dos profissionais, somada aos dados disponíveis na literatura internacional, permitiu elaborar este documento, produzido com o objetivo de reunir e harmonizar as informações disponíveis sobre o tratamento destas doenças graves e progressivas, mas que felizmente hoje são tratáveis, oferecendo novas perspectivas para os pacientes brasileiros afetados por essas condições.

\section{Agradecimentos}

Os autores deste documento agradecem a Liliane Koester por seu apoio logístico, especialmente na organização da reunião dos especialistas que participaram da elaboração deste documento, a qual realizou-se em Caxias do Sul (RS) em 2008. Agradecemos, também, a Márcia Trinfol pela contribuição na redação científica e ao CLADEGEN pelo apoio irrestrito a esta iniciativa.

Suporte Financeiro: 0 presente trabalho recebeu suporte financeiro da Fundação Médica do Rio Grande do Sul, e do GLADEGEN - Centro Latino Americano para o Desenvolvimento da Genética na Medicina.

Conflito de interesse: Todos os autores declararam não haver conflito de interesse no momento que participaram da elaboração deste documento.

\section{SUMMARY}

ENZYME REPLACEMENT THERAPY FOR MUCOPOLYSACCHARIDOSES I, II AND VI: RECOMMENDATIONS FROM A GROUP OF BRAZILIAN F EXPERTS

Mucopolysaccharidoses (MPS) are rare genetic diseases caused by deficiency of specific lysosomal enzymes that affect catabolism of glycosaminoglycans (GAG). Accumulation of GAG in various organs and tissues in MPS patients results in a series of signs and symptoms, producing a multisystemic condition affecting bones and joints, the respiratory and cardiovascular systems and many other organs and tissues, including in some cases, cognitive performance. So far, eleven enzyme defects that cause seven different types of MPS have been identified. Before introduction of therapies to restore deficient enzyme activity, treatment of MPS focused primnarily on prevention and care of complications, still a very important aspect in the management of these patients. In the 80's treatment of MPS with bone marrow transplantation/hematopoietic stem cells transplantation (BMT) HSCT) was proposed and in the 90's, enzyme replacement therapy (ERT), began to be developed and was approved for clinical use in MPS I, II and VI in the first decade of the 21st century. The authors of this paper are convinced that a better future for patients affected by mucopolysaccharidoses depends upon identifying, understanding and appropriately managing the multisystemic manifestations of these diseases. This includes the provision of support measures (which should be part of regular multidisciplinary care of these patients) and of specific therapies. Although inhibition of synthesis of GAG and the recovery of enzyme activity with small molecules also may play a role in the management of MPS, the breakthrough is the currently available intravenous ERT. ERT radically changed the setting for treatment of mucopolysaccharidosis I, II and VI in the last decade., Benefits can even be extended soon to MPS IV A (ERT for this condition is already in clinical development), with prediction for treatment of MPS III A and the cognitive deficit in MPS // by administration of the enzyme directly into the central nervous system (CNS). A large number of Brazilian services, from all regions of the country, already have experience with ERT for MPS I, II and VI. This experience was gained not only by treating patients but also with the participation of some groups in clinical trials involving ERT for these conditions. Summing up the three types of MPS, more than 250 patients have already been treated with ERT in Brazil. The experience of professionals coupled to the data available in international literature, allowed us to elaborate this document, produced with the goal of bringing together and harmonize the information available for the treatment of these severe and progressive diseases, which, fortunately, are now treatable, a situation which bring new perspectives for Brazilian patients, affected by these conditions.

KEY WORDS: Mucopolysaccharidosis I. Mucopolysaccharidosis II. Mucopolysaccharidosis VI. Glycosaminoglycans V. Enzyme replacement therapy.

\section{REFERÊNCIAS}

1. Neufeld EF, Muenzer J. The mucopolysaccharidosis. In: Scriver CR, Beaudet AL, Sly WS, Valle D, editors. The metabolic and molecular bases of inherited disease. New York (NY): McGraw-Hill Co; 2001. p.3421-52.

2. Ard JL Jr, Bekker A, Frempong-Boadu AK. Anesthesia for na adult with mucopolysaccharidosis I. J Clin Anesth. 2005;17(8):624-6.

3. Leistner S, Giugliani R. A useful routine for the biochemical detection and diagnosis of mucopolysaccharidoses. Genet Molec Biol. 1998;21(1):163-7.

4. Krivit W. Allogeneic stem cell transplantation for the treatment of lysosomal and peroxisomal metabolic diseases. Springer Semin Immunopathol. 2004;26(1-2): 119-32.

5. Lange MC, Teive HA, Troiano AR, Bitencourt M, Funke VA, Setúbal DC, et al. Bone marrow transplantation in patients with storage diseases: a developing country experience. Arq Neuropsiquiatr. 2006;64(1):1-4.

6. Kakkis ED, Muenzer J, Tiller GE, Waber L, Belmont J, Passage M, et al. Enzyme Replacement Therapy in Mucopolysaccharidosis I. N Engl J Med. $2001 ; 344(3): 182-8$

7. Kakkis ED, Schuchman E, He X, Wan Q, Kania S, Wiemelt S, et al. Enzyme replacement therapy in feline mucopolysaccharidosis I. Mol Genet Metab. 2001;72(3): 199-208. 
8. Wraith JE, Clarke LA, Beck M, Kolodny EH, Pastores GM, Muenzer J, et al. Enzyme Replacement Therapy for Mucopolysaccharidosis I: a Randomized Double-Blinded, Placebo-Controlled, Multinational Study of Recombinant Human $[$-L-Iduronidase (Laronidase). J Pediatr. 2004;144(5):581-8.

9. Harmatz P, Kramer WG, Hopwood JJ, Simon J, Butensky E, Swiedler SJ, Mucopolysaccharidosis VI Study Group. Pharmacokinetic profile of recombinant human $\mathrm{N}$-acetylgalactosamine 4-sulphatase enzyme replacement therapy in patients with mucopolysaccharidosis VI (Maroteaux-Lamy syndrome): a phase I/II study. Acta Paediatr Suppl. 2005;94(447):61-8.

10. Harmatz P, Ketteridge D, Giugliani R, Guffon N, Teles EL, Miranda MC, et al. Direct comparasion of measures of endurance, mobility, and joint function during enzyme-replacement therapy of Mucopolisaccharidosis VI (MaroteauxLamy Syndrome): results after 48 weeks in a phase 2 open-labem clinical study of recombinant human $\mathrm{N}$-acetylgalactosamine 4-sulfatase. Pediatrics. 2005;115(6):681-9.

11. Wraith JE. The first 5 years of clinical experience with laronidase enzyme replacement therapy for mucopolysaccharidosis I. Expert Opin Pharmacother. 2005;6(3):489-506. 12. Muenzer J, Wraith JE, Beck M, Giugliani R, Harmatz P, Eng CM, et al. A phase II/III clinical study of enzyme replacement therapy with idursulfase in mucopolisaccharidosis II (Hunter syndrome). Genet Med. 2006;8(8):465-73.

13. Muenzer J, Guscsavas-Calikoglu M, Shawn E, Schuetz TJ, Kimura A. A phase I/II clinical trial of enzyme replacemet therapy in mucopolisaccharidosis II (Hunter Syndrome). Mol Genet Metab. 2007;90(3):329-37.

14. Sifuentes M, Doroshow R, Hoft R, Mason G, Walot I, Diament M, et al A follow up study of MPS I patients treated with laronidase enzyme replacement therapy for 6 years. Mol Genet Metab. 2007:90(2):171-80. 15. Wraith JE, Beck M, Lane R, van der Ploeg A, Shapiro E, Xue $Y$, et al. Enzyme replacement therapy in patients who have mucopolysaccharidosis I and are younger than 5 years: results of a multinational study of recombinant human $\mathrm{D-L-iduronidase} \mathrm{(laronidase).} \mathrm{Pediatrics.} \mathrm{2007;120(1):37-46.}$

16. Clarke LA. Idursulfase for the treatment of mucopolysaccharidosis II. Expert Opin Pharmacother. 2008;9(2):311-17

17. Harmatz P, Giugliani R, Schwartz I, Guffon N, Teles E, Sá Miranda M, etal. Long term follow-up of endurance and safety outcomes during enzyme replacement therapy for mucopolysaccharidosis VI: Final results of three clinical studies of recombinant human N-acetylgalactosamine 4-sulfatase. Mol Genet Metab. 2008:94(4):469-75.

18. Clarke LA, Wraith JE, Beck M, Kolodny EH, Pastores GM, Muenzer J, et al Long-term efficacy and safety of laronidase in the treatment of mucopolysacchoaridosis I. Pediatrics. 2009;123(1):229-40.

19. Giugliani R, Muñoz-Rojas MV, Martins AM, Valadares ER, Clarke JT, Góes JE, et al. A dose-optimization trial of laronidase (Aldurazyme $\left({ }^{\circledR}\right)$ in patients with mucopolysaccharidosis I. Mol Genet Metabol. 2009;96(1):13-9.

19. Boelens JJ, Wynn RF, O'meara A, Veys P, Bertrand Y, Souillet G, et al. Outcomes of hematopoietic stem cell transplantation for Hurler's syndrome in Europe: a risk factor analysis for graft failure. Bone Marrow Transplant. 2007;40(3):225-33.

20. Barton NW, Furbish FS, Murray GJ, Garfield M, Brady RO. Therapeutic response to intravenous infusions of glucocerebrosidase in a patient with Gaucher disease. Proc Nat Acad Sci USA. 1990;87(5):1913-6.

21. Biomarin [cited 2009 mar 3]. Biomarin Pharmaceutical Inc. Available from: http://www.bmrn.com/products/aldurazyme.php.

22. Biomarin [cited 2009 mar 9]. Biomarin Pharmaceutical Inc. Available from: http://www.bmrn.com/products/naglazyme.php.
23. Elaprase [cited 2009 mar 3]. Elaprase (idursulfase). Available from; http:// www.elaprase.com

24. FDA. US Food and Drug Administration. Drugs@ FDA. [cited 2009 mar 3]. Available from: http://www.accessdata.fda.gov/scripts/cder/drugsatfda/index. $\mathrm{cfm}$ ?fuseaction $=$ Search. DrugDetails.

25. EMEA. European Medicines Agency. [cited 2009 mar 3]. Available from: http:// www.emea.europa.eu.

26. ANVISA. Agência Nacional de Vigilância Sanitária. [citado 3 mar 2009]. Disponível em: http://www.anvisa.gov.br/.

27. Fuller M, Hopwood JJ, Anson DS. Receptor mediated binding of two glycosylation of $\mathrm{N}$-acetylgalactosamine-4-sulphatase. Biochim Biophys Acta. 1998;1406(3):283-90.

28. Auclair D, Hopwood JJ, Brooks DA, Lemontt JF, Crawley AC. Replacement therapy in Mucopolysaccharidosis type $\mathrm{VI}$ : advatages of early onset of therapy. Mol Genet Metab. 2003;78(3):163-74.

29. Wraith JE, Scarpa M, Beck M, Bodamer AO, De Meirleir L, Guffon N, Lund AM, Malm G, Vand der Ploeg A, Zeman J. Mucopolysaccharidosis type II (hunter syndrome): a clinical review and recommendations for treatment in the era of enzyme replacement therapy. Eur J Pediatr.

30. Giugliani R, Harmatz P, Wraith JE. Management guidelines for mucopolysaccharidosis VI. Pediatrics, 2007; 120 (2):405-18.

31. Harmatz P, Giugliani R, Schwartz I, Guffon N, Teles E, Sá Miranda M, MPS VI Phase 3 Study Group. Enzyme replacement therapy for mucopolysaccharidosis $\mathrm{VI}$ : a phase 3, randomized, double-bind, placebo-controlled, multinational study of recombinant human $\mathrm{N}$-acetylgalactosamine 4-sulfatase (recombinan human arylsulfatase B or rhASB) and follow-on, open-label extension study. J Pediatr. 2006;148(4):533-9.

32. McGill JJ, Inwood AC, Coman DJ, Lipke ML, Skinner J, Morris B. Enzyme replacement therapy for MPS VI with recombinant human $\mathrm{N}$-acetygalactosamine 4-sulfatase (rhASB) from 8 weeks of age - a sibling control study. J Inher Metab Dis. 2006;29:Suppl 1:65.

33. Anbu A, Mercer J, Wraith JE. Effect of discontinuing of laronidase in a patient with mucopolysaccharidosis type I. J Inherit Metab Dis. 2006;29:230-1.

34. Wegrzyn G, Tylki-Szymanska A, Keberek A, COMPLETAR. Rapid deterioration of a patient with mucopolysaccharidosis type I during interruption of enzyme replacement therapy. Am J Med Genet Part A. 2007;143A:1925-7.

35. Kim KH, Decker C, Burton BK. Successful management of difficult infusionassociated reactions in a young patient with mucopolysaccharidosis type $\mathrm{VI}$ receiving recombinant human arysulfatase B (galsulfase [Naglazyme]). Pediatrics. 2008;12(3):714-7.

36. Muñoz-Rojas MV, Vieira T, Costa R, Fagondes S, John A, Jardim LB et al. Intrathecal enzyme replacement therapy in a patient with mucopolysaccharidosis type I and symptomatic cord compression. Am J Med Genet. 2008:146A:2538-44

37. Cox-Brikman J, Timmermans RGM, Wijbrug FA, Donker WE, van de Ploeg AT, Aerts JM et al. Home treatment with enzyme replacement therapy for mucopolysaccharidosis type I is feasible and safe. J Inherit Metab Dis. 2007:30(6):984. Epub 2007 sep 21.

38. Pereira VG, Matins AM, Micheletti C, COMPLETAR. Mutational and oxidative stress analysis in patients with mucopolysaccharidosis type I undergoing enzyme replacement therapy. Clin Chim Acta. 2008;387(1-2):75-9.

39. Randall DR, Sinclair GB, Colobong KE, Hetty E, Clarke LA. Heparin Cofactor II-thrombin complex in MPS I: a biomarker of MPS disease. Mol Genet Metab. 2006;88(3):235-43. 